\title{
DRAFT: THE EFFECTS OF TRAILER TOWING ON THE DYNAMICS OF A LANE-KEEPING CONTROLLER
}

\author{
Illés Vörös \\ Department of Applied Mechanics \\ Budapest University of Technology \\ and Economics \\ Budapest, $\mathrm{H}-1111$ \\ Hungary \\ Email: illes.voros@mm.bme.hu
}

\author{
Dénes Takács \\ MTA-BME Research Group on \\ Dynamics of Machines and Vehicles \\ Budapest, $\mathrm{H}-1111$ \\ Hungary \\ takacs@mm.bme.hu
}

\begin{abstract}
The main question this paper aims to address is whether a lane-keeping controller can serve as a safety feature in order to increase handling and stability of car-trailer combinations. The derivation of a nonholonomic car-trailer model is provided and its open-loop stability is analyzed. A look-ahead based lanekeeping controller is applied to the system, and a comparison is made between the closed-loop stability of an individual car and a car-trailer setup. The critical speed of the vehicle combination is shown as a function of various trailer parameters, with and without the lane-keeping controller enabled. A modified version of the controller is also tested, utilizing feedback of the trailer states. This ensures that the trailer is also kept at the center of the lane, but no improvement was found in terms of stable trailer configurations.
\end{abstract}

\section{INTRODUCTION}

A recent analysis of accident data showed that a large part of road accidents could have been prevented or their severity reduced with the use of certain driver assistance systems [1]. Therefore this paper investigates whether a lane-keeping controller in the towing vehicle can be used as a safety feature to help stabilize a car-trailer system. Unlike traditional trailer stabilization techniques that rely on individual braking of the trailer wheels, a lane-keeping controller can only indirectly control the motion of the trailer through the steering angle of the towing vehicle. Despite this strong limitation, the lane-keeping controller might still prove useful in stabilizing trailers that are not equipped with additional safety features. Another important goal of this paper is to explore how the dynamics of a car with lanekeeping control change when towing a trailer.

The dynamics of trailers have already been studied extensively in the literature, since as early as the 1930s - see the references in [2]. Depending on the use case, lower degree of freedom $[3,4]$, as well as more comprehensive mathematical models $[5,6]$ have both been developed. A nonlinear analysis of truck and trailer systems can be found in [7], while notable experimental studies include [8] and [9].

In terms of stability and handling characteristics, several solutions have been proposed relying on active rear wheel steering of the towing vehicle $[10,11]$, active trailer steering or braking $[12,13]$, or active braking of both the towing vehicle and the trailer [14]. A solution that does not require actuators on the trailer is using the brake system of the towing vehicle to improve the stability of the vehicle combination [15]. In this paper, active steering of the front wheels of the towing vehicle is considered, through the utilization of a lane-keeping controller.

The rest of the paper is organized as follows: first, a detailed derivation of the governing equations of the car and trailer model is presented. The dynamics of the open-loop system are then analyzed to check how certain trailer parameters influence 
stability as a function of speed. Afterwards, a lane-keeping controller is introduced in the towing vehicle: it is examined how the presence of the trailer influences the stability properties of lane keeping compared to a car only setup. Then it is also checked how the dynamics of the open-loop car and trailer system change due to the controller. Finally, the lane-keeping control law is extended with the feedback of trailer states to see if there is any benefit in terms of stable parameter combinations.

\section{MECHANICAL MODEL OF THE CAR-TRAILER SYSTEM}

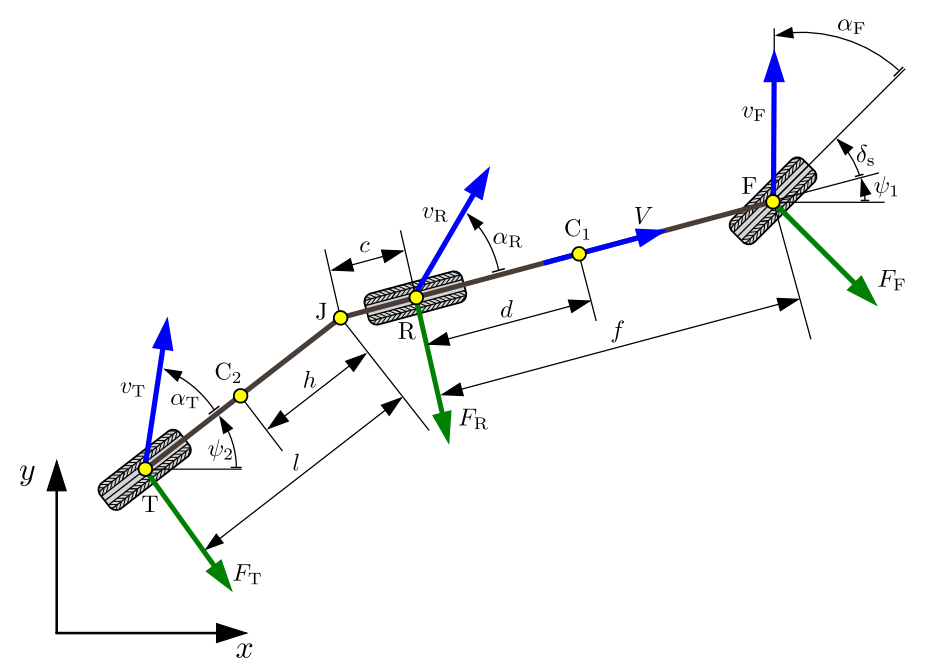

FIGURE 1. The single track model of the car and trailer system.

The in-plane motion of the car-trailer system is described using a simplified single-track model (see Fig. 1). This means that the tire contact patches are summarized axle-wise in points $\mathrm{F}$ (car front axle), $\mathrm{R}$ (car rear axle) and $\mathrm{T}$ (trailer axle). The center of gravity of the car and the trailer is denoted by $C_{1}$ and $\mathrm{C}_{2}$, respectively, while $\mathrm{J}$ refers to the hitch point. The coordinates of point $\mathrm{R}$ in the global reference frame are denoted by $x$ and $y$, and the orientation of the car and the trailer are $\psi_{1}$ and $\psi_{2}$. The steering angle of the car is denoted by $\delta_{\mathrm{s}}$. The geometrical and inertial parameters and their notations are listed in Table 1.

The longitudinal velocity of the car is assumed to remain constant $(V)$, which can be translated into the following kinematic constraint:

$$
\dot{x} \cos \psi_{1}+\dot{y} \sin \psi_{1}=V .
$$

This makes the system nonholonomic, therefore we use the Gibbs-Appell-method [16] to derive its equations of motion.
Since the system includes only one kinematic constraint equation, but the number of state variables is four, three so-called pseudo velocities need to be defined in order to proceed. These can be chosen intuitively, but they need to satisfy the constraint equations. In our case, let us make the first pseudo velocity $\left(\sigma_{1}\right)$ the lateral velocity component of point $\mathrm{R}$, while the other two will be the yaw rate of the car $\left(\sigma_{2}\right)$ and the trailer $\left(\sigma_{3}\right)$. The definitions of the pseudo velocities along with the kinematic constraint (1) can be solved for the derivatives of the states:

$$
\begin{aligned}
\dot{x} & =V \cos \psi_{1}-\sigma_{1} \sin \psi_{1}, \\
\dot{y} & =V \sin \psi_{1}+\sigma_{1} \cos \psi_{1}, \\
\dot{\psi}_{1} & =\sigma_{2}, \\
\dot{\psi}_{2} & =\sigma_{3} .
\end{aligned}
$$

The rest of the system dynamics (i.e. how the pseudovelocities $\sigma_{i}$ change in time) are determined by the Gibbs-Appell equations

$$
\frac{\partial G}{\partial \dot{\sigma}_{i}}=\Gamma_{i}, \quad i=1,2,3
$$

The so-called energy of acceleration $G$ in the left-hand side of Eqn. (3) can be calculated as

$$
G=\frac{1}{2} m_{1} a_{\mathrm{C}_{1}}^{2}+\frac{1}{2} J_{1} \ddot{\psi}_{1}^{2}+\frac{1}{2} m_{2} a_{\mathrm{C}_{2}}^{2}+\frac{1}{2} J_{2} \ddot{\psi}_{2}^{2}+\ldots
$$

for the in-plane motion of the car and trailer. Equation (4) is not the complete form of the energy of acceleration, but the remaining terms' derivatives in Eqn. (3) are zero, therefore they do not affect our further calculations. The accelerations of the centers of gravity $a_{\mathrm{C}_{1}}$ and $a_{\mathrm{C}_{2}}$, as well as the angular accelerations $\ddot{\psi}_{1}$ and $\ddot{\psi}_{2}$ can be expressed as a function of the pseudo-velocities and their time derivatives. Substituting them in $G$ leads to the derivatives

$$
\begin{aligned}
\frac{\partial G}{\partial \dot{\sigma}_{1}} & =\left(m_{1}+m_{2}\right) V \sigma_{2}+m_{1}\left(\dot{\sigma}_{1}+d \dot{\sigma}_{2}\right)+ \\
& -m_{2}\left(h \sigma_{3}^{2} \sin \left(\psi_{1}-\psi_{2}\right)-\dot{\sigma}_{1}+c \dot{\sigma}_{2}+h \dot{\sigma}_{3} \cos \left(\psi_{1}-\psi_{2}\right)\right) \\
\frac{\partial G}{\partial \dot{\sigma}_{2}} & =\left(d m_{1}-c m_{2}\right) V \sigma_{2}+d m_{1} \dot{\sigma}_{1}+\left(J_{1}+d^{2} m_{1}\right) \dot{\sigma}_{2}+ \\
& +c m_{2} \sigma_{3}^{2}\left(h \sin \left(\psi_{1}-\psi_{2}\right)-\dot{\sigma}_{1}+c \dot{\sigma}_{2}+h \dot{\sigma}_{3} \cos \left(\psi_{1}-\psi_{2}\right)\right) \\
\frac{\partial G}{\partial \dot{\sigma}_{3}} & =-h m_{2}\left(\left(V \cos \left(\psi_{1}-\psi_{2}\right)-\sigma_{1} \sin \left(\psi_{1}-\psi_{2}\right)\right) \sigma_{2}+\right. \\
& \left.+c \sigma_{2}^{2} \sin \left(\psi_{1}-\psi_{2}\right)+\left(\dot{\sigma}_{1}-c \dot{\sigma}_{2}\right) \cos \left(\psi_{1}-\psi_{2}\right)\right)+ \\
& +\left(J_{2}+h^{2} m_{2}\right) \dot{\sigma}_{3} .
\end{aligned}
$$


The terms $\Gamma_{i}$ in the right-hand side of Eqn. (3) are the socalled pseudo-forces that can be derived from the virtual power of the active forces. Since active forces emerge only at the tires in our model, the virtual power is of the form

$$
\delta P=\mathbf{F}_{\mathrm{F}} \delta \mathbf{v}_{\mathrm{F}}+\mathbf{F}_{\mathrm{R}} \delta \mathbf{v}_{\mathrm{R}}+\mathbf{F}_{\mathrm{T}} \delta \mathbf{v}_{\mathrm{T}},
$$

where $\delta$ indicates the virtual quantities. We consider only the lateral component of the tire forces in our analysis, therefore the direction of the force vectors in Eqn. (6) is determined by the orientation of the wheels:

$$
\begin{aligned}
& \mathbf{F}_{\mathrm{F}}=\left[\begin{array}{c}
F_{\mathrm{F}, x} \\
F_{\mathrm{F}, y} \\
0
\end{array}\right]=\left[\begin{array}{c}
F_{\mathrm{F}}^{\text {lat }} \sin \left(\psi_{1}+\delta_{\mathrm{s}}\right) \\
-F_{\mathrm{F}}^{\text {lat }} \cos \left(\psi_{1}+\delta_{\mathrm{s}}\right) \\
0
\end{array}\right], \\
& \mathbf{F}_{\mathrm{R}}=\left[\begin{array}{c}
F_{\mathrm{R}, x} \\
F_{\mathrm{R}, y} \\
0
\end{array}\right]=\left[\begin{array}{c}
F_{\mathrm{R}}^{\text {lat }} \sin \psi_{1} \\
-F_{\mathrm{R}}^{\text {lat }} \cos \psi_{1} \\
0
\end{array}\right], \\
& \mathbf{F}_{\mathrm{T}}=\left[\begin{array}{c}
F_{\mathrm{T}, x} \\
F_{\mathrm{T}, y} \\
0
\end{array}\right]=\left[\begin{array}{c}
F_{\mathrm{T}}^{\text {lat }} \sin \psi_{2} \\
-F_{\mathrm{T}}^{\text {lat }} \cos \psi_{2} \\
0
\end{array}\right] .
\end{aligned}
$$

Assuming a linear tire characteristic, the magnitudes $F_{i}^{\text {lat }}$ of the side forces are proportional to the side slip angles $\alpha_{i}$ :

$$
F_{i}^{\text {lat }}=C_{i} \alpha_{i}, \quad i \in\{\mathrm{F}, \mathrm{R}, \mathrm{T}\}
$$

where $C_{i}$ denotes the cornering stiffness of the respective tires. The slip angles can be expressed as

$$
\begin{aligned}
& \alpha_{\mathrm{F}}=\arctan \left(\frac{\dot{y}+f \dot{\psi}_{1} \cos \psi_{1}}{\dot{x}-f \dot{\psi}_{1} \sin \psi_{1}}\right)-\psi_{1}-\delta_{\mathrm{s}}, \\
& \alpha_{\mathrm{R}}=\arctan \left(\frac{\dot{y}}{\dot{x}}\right)-\psi_{1}, \\
& \alpha_{\mathrm{T}}=\arctan \left(\frac{\dot{y}-c \dot{\psi}_{1} \cos \psi_{1}-l \dot{\psi}_{2} \cos \psi_{2}}{\dot{x}+c \dot{\psi}_{1} \sin \psi_{1}+l \dot{\psi}_{2} \sin \psi_{2}}\right)-\psi_{2} .
\end{aligned}
$$

Substituting the force components according to Eqn. (7) as well as the virtual velocity vectors of the respective points into Eqn. (6), the virtual power can be written as

$$
\begin{aligned}
\delta P & =\delta \sigma_{1}\left(\left(F_{\mathrm{F}, y}+F_{\mathrm{R}, y}+F_{\mathrm{T}, y}\right) \cos \psi_{1}-\left(F_{\mathrm{F}, x}+F_{\mathrm{R}, x}+F_{\mathrm{T}, x}\right) \sin \psi_{1}\right)+ \\
& +\delta \sigma_{2}\left(\left(f F_{\mathrm{F}, y}-c F_{\mathrm{T}, y}\right) \cos \psi_{1}+\left(-f F_{\mathrm{F}, x}+c F_{\mathrm{T}, x}\right) \sin \psi_{1}\right)+ \\
& +\delta \sigma_{3} l\left(-F_{\mathrm{T}, y} \cos \psi_{2}+F_{\mathrm{T}, x} \sin \psi_{2}\right),
\end{aligned}
$$

taking into account that the virtual power of the kinematic constraint is zero. The coefficients of $\delta \sigma_{i}$ from the virtual power form the pseudo-forces:

$$
\begin{aligned}
& \Gamma_{1}=\left(F_{\mathrm{F}, y}+F_{\mathrm{R}, y}+F_{\mathrm{T}, y}\right) \cos \psi_{1}-\left(F_{\mathrm{F}, x}+F_{\mathrm{R}, x}+F_{\mathrm{T}, x}\right) \sin \psi_{1}, \\
& \Gamma_{2}=\left(f F_{\mathrm{F}, y}-c F_{\mathrm{T}, y}\right) \cos \psi_{1}+\left(-f F_{\mathrm{F}, x}+c F_{\mathrm{T}, x}\right) \sin \psi_{1}, \\
& \Gamma_{3}=l\left(F_{\mathrm{T}, x} \sin \psi_{2}-F_{\mathrm{T}, y} \cos \psi_{2}\right) .
\end{aligned}
$$

The remaining equations of motion can be reached by equating the elements of Eqn. (5) with Eqn. (13), according to Eqn. (3). These equations can be solved for the time derivatives of the pseudo-velocities, and the force components can be substituted according to Eqn. (7) and (8). Coupled with the formulas for the derivatives of the state variables (Eqn. (2)), these constitute the governing equations of the car and trailer system in first order form.

\section{OPEN-LOOP STABILITY}

In order to determine the stability of straight-line motion without control action, the equations of motion are first transformed into second-order form. To do this, we take the linearized form of the definition of the pseudo-velocities:

$$
\begin{aligned}
& \sigma_{1}=\dot{y}-V \psi_{1}, \\
& \sigma_{2}=\dot{\psi}_{1}, \\
& \sigma_{3}=\dot{\psi}_{2},
\end{aligned}
$$

since we are only interested in the small oscillations around the straight-line motion of the vehicle. The above are substituted into the last three governing equations, while the remaining trigonometric nonlinearities are linearized around zero, and any second or higher order term is neglected. This leads to the following set of linear, second-order differential equations:

$$
\begin{aligned}
& \left(m_{1}+m_{2}\right) \ddot{y}+\left(d m_{1}-c m_{2}\right) \ddot{\psi}_{1}-h m_{2} \ddot{\psi}_{2}= \\
& \quad=F_{\mathrm{F}, y}+F_{\mathrm{R}, y}+F_{\mathrm{T}, y}, \\
& \left(d m_{1}-c m_{2}\right) \ddot{y}+\left(J_{1}+d^{2} m_{1}+c^{2} m_{2}\right) \ddot{\psi}_{1}+c h m_{2} \ddot{\psi}_{2}= \\
& \quad=F_{\mathrm{F}, y} f-c F_{\mathrm{T}, y}, \\
& \left(J_{2}+h^{2} m_{2}\right) \ddot{\psi}_{2}-h m_{2}\left(\ddot{y}-c \ddot{\psi}_{1}\right)=-l F_{\mathrm{T}, y} .
\end{aligned}
$$

After taking the similarly linearized form of the tire side force components (while setting the steering angle $\delta_{\mathrm{s}}$ to zero) the system can be transformed into the matrix form

$$
\mathbf{M} \ddot{\mathbf{q}}+\mathbf{C} \dot{\mathbf{q}}+\mathbf{K q}=\mathbf{0}
$$


with the corresponding state vector $\mathbf{q}=\left[\begin{array}{lll}y & \psi_{1} & \psi_{2}\end{array}\right]^{\mathrm{T}}$ and coefficient matrices

$$
\begin{aligned}
\mathbf{M} & =\left[\begin{array}{ccc}
m_{1}+m_{2} & m_{1} d-m_{2} c & -m_{2} h \\
m_{1} d-m_{2} c & J_{1}+m_{1} d^{2}+m_{2} c^{2} & m_{2} c h \\
-m_{2} h & m_{2} c h & J_{2}+m_{2} h^{2}
\end{array}\right], \\
\mathbf{C} & =\frac{1}{V}\left[\begin{array}{ccc}
C_{\mathrm{F}}+C_{\mathrm{R}}+C_{\mathrm{T}} & f C_{\mathrm{F}}-c C_{\mathrm{T}} & -l C_{\mathrm{T}} \\
f C_{\mathrm{F}}-c C_{\mathrm{T}} & f^{2} C_{\mathrm{F}}+c^{2} C_{\mathrm{T}} & c l C_{\mathrm{T}} \\
-l C_{\mathrm{T}} & c l C_{\mathrm{T}} & l^{2} C_{\mathrm{T}}
\end{array}\right], \\
\mathbf{K} & =\left[\begin{array}{ccc}
0-C_{\mathrm{F}}-C_{\mathrm{R}}-C_{\mathrm{T}} \\
0 & -f C_{\mathrm{F}} & c C_{\mathrm{T}} \\
0 & 0 & l C_{\mathrm{T}}
\end{array}\right] .
\end{aligned}
$$

The characteristic equation of the open-loop system is therefore

$$
D_{1}(\lambda):=\operatorname{det}\left(\lambda^{2} \mathbf{M}+\lambda \mathbf{C}+\mathbf{K}\right)=0,
$$

where $\lambda \in \mathbb{C}$ denotes the characteristic exponent. Note that the above equation has two zero roots corresponding to the position and orientation of the vehicle in the plane, which has no influence on the stability of straight-line motion. Therefore it is sufficient to consider the equation $D_{1}(\lambda) / \lambda^{2}=0$. Stability is ensured if all $\lambda_{i}$ roots of this equation reside on the left half of the complex plane. This can be verified using e.g. the Routh-Hurwitz criterion [17].

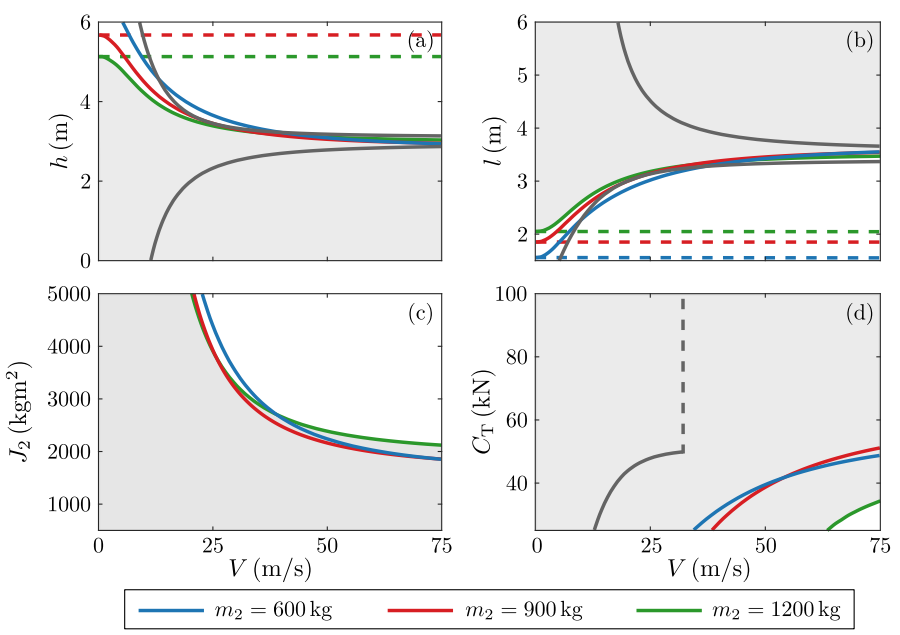

FIGURE 2. Stability charts of various trailer parameters and speed for the open-loop car-trailer combination. Dashed lines represent boundaries of static loss of stability. The shaded areas show the stable regions for the trailer mass $m_{2}=1200 \mathrm{~kg}$.

The stability maps in Fig. 2 show the stable domains of

\begin{tabular}{|c|c|c|}
\hline Parameter & Notation & Value \\
\hline Car wheelbase & $f$ & $2.7 \mathrm{~m}$ \\
\hline $\begin{array}{l}\text { Distance between car rear axle } \\
\text { and center of gravity }\end{array}$ & $d$ & $1.35 \mathrm{~m}$ \\
\hline $\begin{array}{l}\text { Distance between car rear axle } \\
\text { and hitch point }\end{array}$ & $c$ & $0.75 \mathrm{~m}$ \\
\hline Trailer length & $l$ & $3.5 \mathrm{~m}$ \\
\hline $\begin{array}{l}\text { Distance between trailer center } \\
\text { of gravity and hitch point }\end{array}$ & $h$ & $3 \mathrm{~m}$ \\
\hline Mass of car & $m_{1}$ & $1430 \mathrm{~kg}$ \\
\hline Mass of trailer & $m_{2}$ & $900 \mathrm{~kg}$ \\
\hline Yaw moment of inertia of car & $J_{1}$ & $2500 \mathrm{kgm}^{2}$ \\
\hline Yaw moment of inertia of trailer & $J_{2}$ & $2000 \mathrm{kgm}^{2}$ \\
\hline $\begin{array}{l}\text { Cornering stiffness of car front tire } \\
\text { (without trailer) }\end{array}$ & $C_{\mathrm{F}}^{0}$ & $45 \mathrm{kN}$ \\
\hline $\begin{array}{l}\text { Cornering stiffness of car rear tire } \\
\text { (without trailer) }\end{array}$ & $C_{\mathrm{R}}^{0}$ & $60 \mathrm{kN}$ \\
\hline Cornering stiffness of trailer tire & $C_{\mathrm{T}}$ & $45 \mathrm{kN}$ \\
\hline Longitudinal velocity of car & $V$ & $20 \mathrm{~m} / \mathrm{s}$ \\
\hline Time delay & $\tau$ & $0.5 \mathrm{~s}$ \\
\hline
\end{tabular}
trailer parameters as a function of speed for the nominal param-
TABLE 1. Car and Trailer Parameters Used in Stability Maps (Unless Noted Otherwise)

eter values listed in Table 1. It was taken into account that attaching a trailer changes the wheel load distribution of the towing vehicle, thereby affecting the cornering stiffness of its tires. Thus the nominal values $C_{\mathrm{F}}^{0}$ and $C_{\mathrm{R}}^{0}$ were multiplied by $F_{i, z} / F_{i, z}^{0}$ $(i \in\{\mathrm{F}, \mathrm{R}\}$ ), i.e. the ratio of the corresponding axle load with and without the trailer. It should also be kept in mind that the tire forces might saturate at higher speeds, which is not taken into account in the applied linear tire model.

Figure 2 (a) shows that it is not desirable to place the center of gravity too far behind the trailer axle (a trailer length of $l=3.5 \mathrm{~m}$ was used in the plot), especially at higher speeds. When increasing $h$, there exists a physical limit where the rear wheels of the towing vehicle lose contact with the ground. This limit is represented by the horizontal lines in the figure, which correspond to a zero root of the characteristic equation (18) (static loss of stability). If the effect of wheel load distribution on the cornering stiffnesses is not taken into account (dark gray lines in the figure), then this stability limit does not show up. On the other hand, a different stability boundary does show up at lower $h$ values, limiting the stable values of $h$ to a narrow band at higher speeds (as reported in e.g. [7] and [18]). A possible explanation as to why this stability limit cannot be seen when adjusting the value of $C_{\mathrm{F}}$ and $C_{\mathrm{R}}$ is that the additional load on the rear axle makes the vehicle more understeer, which has a stabilizing effect. 
Figure 2 (b) shows that longer trailers are generally more stable, but an additional stability boundary can emerge here as well, if the cornering stiffness values are kept constant. The horizontal lines at low values of $l$ show the limit where the wheel load at the rear axle of the towing vehicle reaches zero (since the center of gravity position of the trailer is not adjusted when changing $l$ ).

According to panel (c), large moments of inertia of the trailer can make the system unstable at higher speeds. The value of $J_{2}$, however, does not influence the wheel load distribution of the towing vehicle.

Finally, the stability map in panel (d) shows that the cornering stiffness of the trailer tire has a stabilizing effect. It was shown in [19] that a vertical stability boundary may also occur at sufficiently high speeds, corresponding to a static loss of stability. This appears if the additional weight of the trailer makes the towing vehicle more oversteer. Since our original choice of parameters involves an understeering vehicle and the center of gravity of the trailer is close to its axle, this effect is shown for a different set of parameters in Fig. 2 (d) in dark gray.

\section{LANE-KEEPING CONTROL}

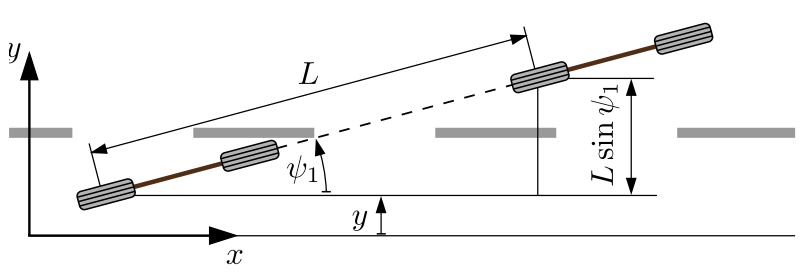

FIGURE 3. Illustration of the look-ahead control scheme.

So far, only the open-loop dynamics of the car and trailer system were considered. In this section, the towing vehicle is equipped with a lane keeping controller that aims to keep the vehicle at the center of the lane. Without loss of generality, we consider a straight-line reference path along the $x$ axis. A simple look-ahead control law is employed, which takes the expected lateral deviation from the reference path at a distance $L$ ahead of the car, assuming that the direction of the vehicle will remain the same (see Fig. 3). The steering angle is generated by feeding back this expected lateral error value through the proportional gain $P_{y}$ :

$$
\delta_{\mathrm{s}}(t)=-P_{y}\left(y(t-\tau)+L \sin \psi_{1}(t-\tau)\right),
$$

where the feedback delay $\tau$ (including effects such as sensor and communication delays, computational time and actuator dynamics) is considered explicitly.

Controlling the lateral position of the vehicle means that its position and orientation in the plane are no longer irrelevant. The equation of $\dot{x}$ from Eq. (2), however, can still be decoupled, since we have chosen the $x$ axis as the reference path. Therefore the state vector in this case is $\mathbf{x}=\left[\begin{array}{llllll}y & \psi_{1} & \psi_{2} & \sigma_{1} & \sigma_{2} & \sigma_{3}\end{array}\right]^{\mathrm{T}}$ where the $\mathbf{x}=\mathbf{0}$ equilibrium represents straight-line motion along the reference. In order to investigate the stability properties, the equations of motion are linearized around this equilibrium, leading to the state space representation

$$
\dot{\mathbf{x}}(t)=\mathbf{A x}(t)+\mathbf{B u}(t-\tau),
$$

where the input $\mathbf{u}(t)=\delta_{\mathrm{s}}(t)$ is the steering angle, $\mathbf{A}$ is a six-bysix matrix and $\mathbf{B}$ is a six-element column vector. Note that the controllability matrix of $\mathbf{A}$ and $\mathbf{B}$ has full rank. The linearized form of control law (19) leads to

$$
\mathbf{u}(t)=-P_{y} y(t)-P_{y} L \psi_{1}(t)
$$

which can be written in the following matrix form:

$$
\mathbf{u}(t)=\hat{\mathbf{K}} \mathbf{x}(t)=\left[\begin{array}{llllll}
-P_{y}-P_{y} L & 0 & 0 & 0 & 0
\end{array}\right] \mathbf{x}(t) .
$$

Using the above notations, the characteristic equation of the time delay system is

$$
D_{2}(\lambda):=\operatorname{det}\left(\lambda \mathbf{I}-\mathbf{A}-\mathbf{B} \hat{\mathbf{K}} \mathrm{e}^{-\lambda \tau}\right)=0
$$

The stability properties of the closed-loop system are analyzed in the next section.

\section{Comparison of car only and car-trailer setups}

The control law (19) is meant to ensure stable path following of a car, but it is perfectly reasonable to assume that a trailer might be attached to the car at some point. In this section, it is examined how towing a trailer influences the stability of the closed-loop system.

Due to the presence of time delay, the characteristic equation (23) is transcendental, with infinitely many roots. In the literature, there exists a wide range of different methods to investigate the stability of such delay differential equations [20-22]. We use the D-subdivision method with the help of the multidimensional bisection method [23] to determine the stability boundaries (where a characteristic root crosses the imaginary axis) in 

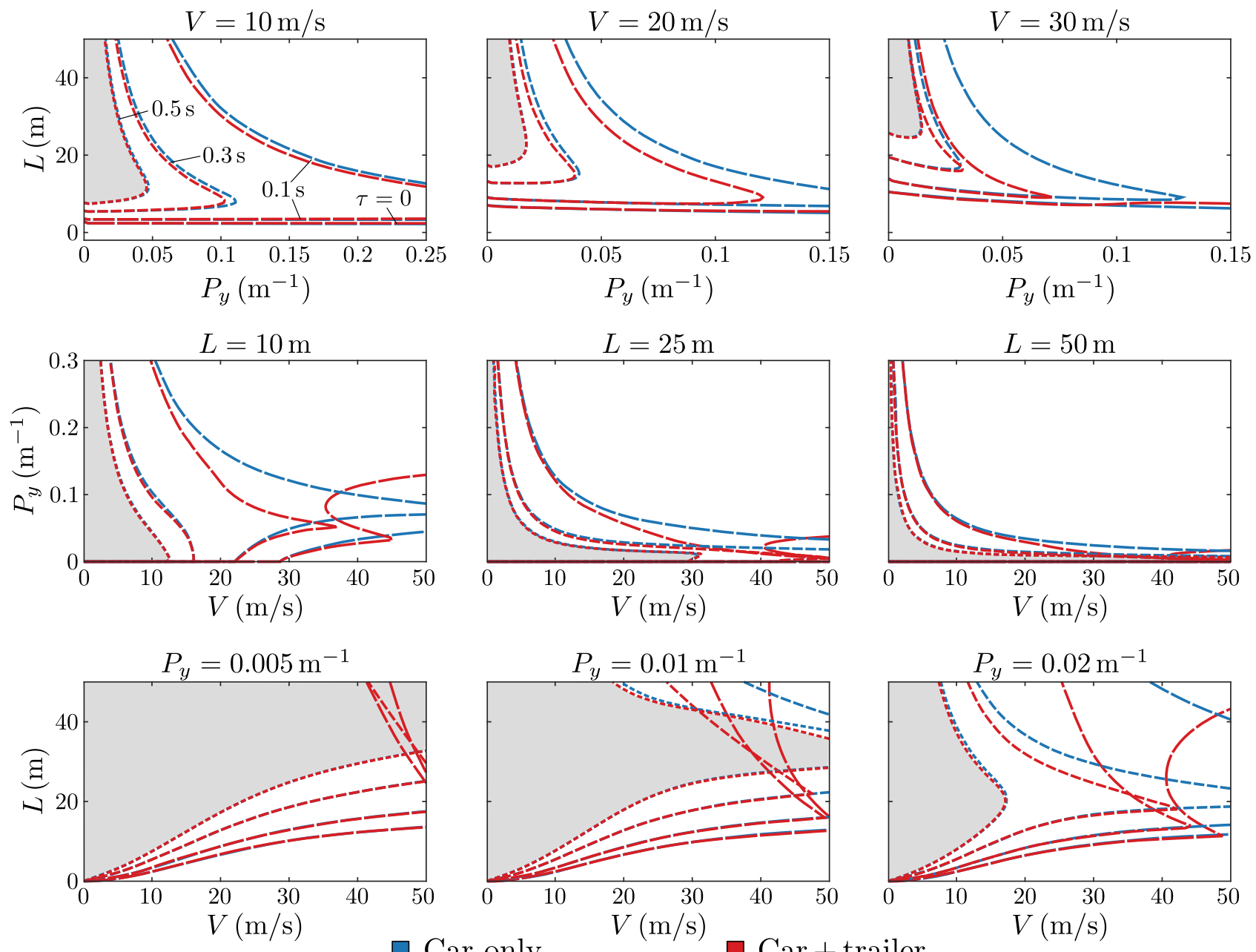

FIGURE 4. Boundaries of stable parameter domains of car only (blue) and car-trailer (red) configurations for various amounts of time delay. The filled area shows the stable domain of the car-trailer combination for $\tau=0.5 \mathrm{~s}$.

different parameter planes. Next, the semi-discretization method [22] is used to verify which domains correspond to zero unstable roots.

The stable parameter domains in terms of control gains and vehicle speed are shown in Fig. 4. The configurations of an individual car and the same car towing a trailer are both plotted for comparison. Additionally, the destabilizing effect of increasing time delay can also be seen. For the governing equations of the car only setup, the reader is referred to [24].

For a given pair of vehicle speed and time delay, there exists a minimum value of $L$, below which the system is unstable. Increasing either $V$ or $\tau$ both increases this minimum distance. It is interesting to note that the maximum allowable look-ahead distance is lower when the trailer is attached, while there is barely any difference in terms of the minimum of $L$. The difference between the car only and the car-trailer configurations is more pronounced at higher speeds. In general, attaching the trailer reduces the stable parameter domains, but increasing the delay value mitigates the difference. In other words, if there is enough time delay in the system, attaching a trailer causes negligible difference in terms of stable parameter domains.

\section{Effects on trailer stability}

Next, we look at how the presence of the lane-keeping controller changes the open-loop stability of the car-trailer system. Figure 5 (a) shows the stability map in the $V-h$ plane with the lane-keeping controller enabled, for various amounts of feedback delay. The control gains were selected with the help of semi-discretization: using a fine enough mesh in the plane of $P_{y}$ and $L$, the optimal control gains in terms of damping factor were determined for the parameter values $V=20 \mathrm{~m} / \mathrm{s}$ and $h=3 \mathrm{~m}$, considering a time delay of $\tau=0.5 \mathrm{~s}$. The resulting control gains 
are $P_{y}=0.0043 \mathrm{~m}^{-1}$ and $L=54.075 \mathrm{~m}$. It can be seen that even in the delay-free case, it is not possible to stabilize previously unstable parameter combinations, and the presence of time delay further reduces the stable domain. A possible explanation for this is that specifying the $y$ coordinate leads to a stricter definition of stability. Earlier, stable straight-line motion regardless of the direction was enough for the system to be considered stable, but now it is also required to specifically follow the $x$ axis. Nevertheless, as long as the delay is not too large, the lane-keeping controller does not change significantly the physically relevant part of the stability map.
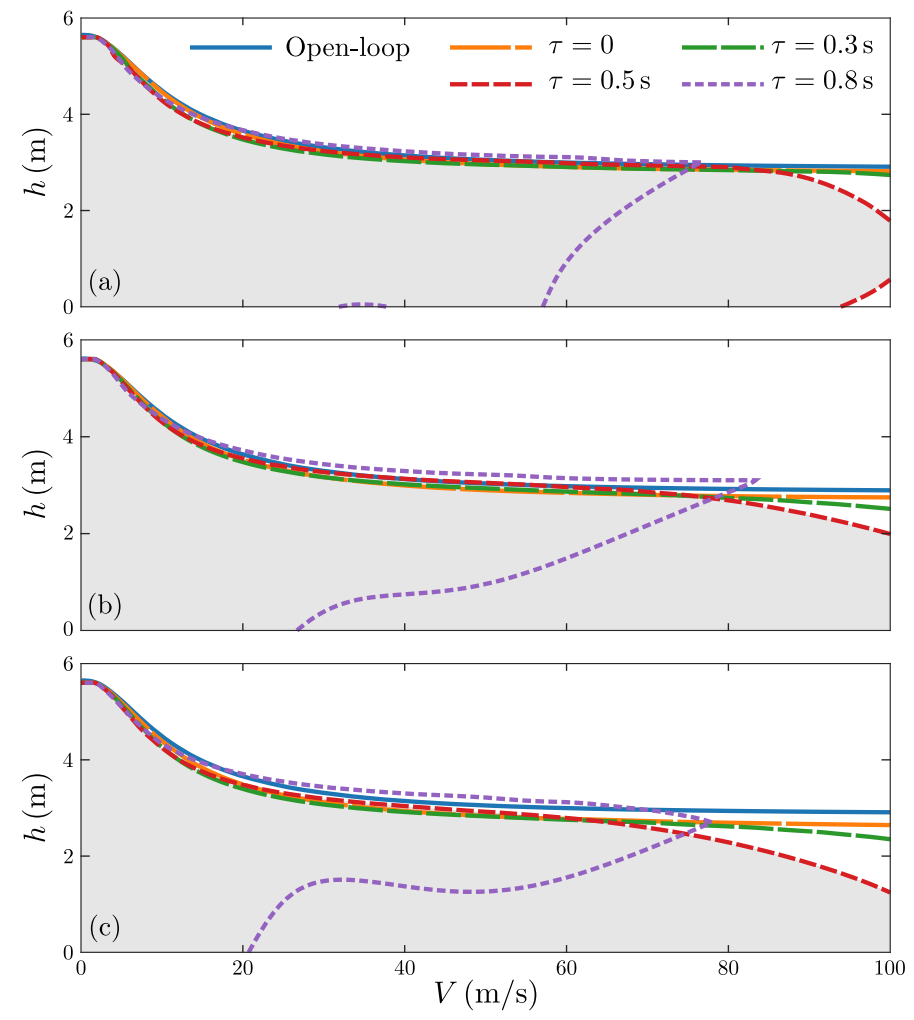

FIGURE 5. Stability boundaries in the $V-h$ plane for the three different control laws: (a) original lane keeping controller, Eqn. (19); (b) additional feedback of trailer yaw angle, Eqn. (24); (c) additional feedback of trailer yaw angle and yaw rate, Eqn. (25). The shaded area shows the stable region for $\tau=0.5 \mathrm{~s}$.

Since control law (19) uses no information regarding the trailer states, it might be possible to improve it by taking into account the orientation of the trailer. Therefore, in the following, modified version of the controller, the yaw angle of the trailer is also fed back through the proportional term $P_{\psi_{2}}$ :

$$
\delta_{\mathrm{S}}(t)=-P_{y}\left(y(t-\tau)+L \sin \psi_{1}(t-\tau)\right)-P_{\psi_{2}} \psi_{2}(t-\tau) .
$$

This modifies the control objective to ensure that the trailer is also kept at the center of the lane, which in itself is beneficial from a safety point of view. The optimal control gains were recalculated using the previously mentioned method, leading to $P_{y}=0.004 \mathrm{~m}^{-1}, L=48.667 \mathrm{~m}$ and $P_{\psi_{2}}=0.026$. The stability chart in Fig. 5 (b) shows that the additional feedback term does not change the stable region significantly, only at high vehicle speeds or large delay values. Therefore as a further extension of the original control law, we also feed back the yaw rate of the trailer $\dot{\psi}_{2}=\sigma_{3}$ :

$$
\begin{aligned}
\delta_{\mathrm{s}}(t)= & -P_{y}\left(y(t-\tau)+L \sin \psi_{1}(t-\tau)\right)+ \\
& -P_{\psi_{2}} \psi_{2}(t-\tau)-P_{\sigma_{3}} \sigma_{3}(t-\tau)
\end{aligned}
$$

The reasoning behind this is that the feedback of a velocity related term might have a dampening effect, which could help stabilize the system. The corresponding control gains are $P_{y}=$ $0.0047 \mathrm{~m}^{-1}, L=44.86 \mathrm{~m}, P_{\psi_{2}}=0.0367$ and $P_{\sigma_{3}}=0.008 \mathrm{~s}$, while the stability chart is shown in Fig. 5 (c). Unfortunately, this control configuration clearly performs worse than the other two in terms of stable parameter combinations, especially at larger feedback delay values. Note, however, that for the largest delay value, $\tau=0.8 \mathrm{~s}$, a small part of the stable domain is outside of the openloop stability boundary. Because of the very small size of this additional stable area, it carries little practical significance due to robustness issues, but this could be an example of the so-called stabilizing effect of feedback delay [25].

It should be noted that the control gains were optimized for $V=20 \mathrm{~m} / \mathrm{s}, h=3 \mathrm{~m}$ and $\tau=0.5 \mathrm{~s}$. In order to stabilize the vehicle at increasing speed or time delay values, the gain $P_{y}$ should be decreased while the look-ahead distance should be increased. Therefore with proper tuning, it is possible to increase the critical speed (compared to what is shown in the stability chart) for a given value of $h$ even for larger delays. However, choosing smaller $P_{y}$ values means that the response will be slower, while increasing the look-ahead distance might not be feasible on curvy roads or due to the limitations of the vision system. A possible solution could be to adjust $L$ according to the vehicle speed. In case of a linear relationship, this would technically mean that the look-ahead point is defined in terms of time (the so-called preview time) instead of distance. Some preliminary results show that in this case, the preview time must be larger than the feedback delay, which is in accordance with Fig. 4, where we showed that zero look-ahead does not stabilize the system.

Figure 6 shows a comparison of the three controller variations (for $\tau=0.5 \mathrm{~s}$ ) and the open-loop system in terms of various trailer parameters. It is interesting to note that in most cases, the original look-ahead controller provides the best performance, while the additional feedback terms only reduce the stable areas. It should still be noted that the shape of the stable domains highly depends on the tuning of the controller, and the control gains here 

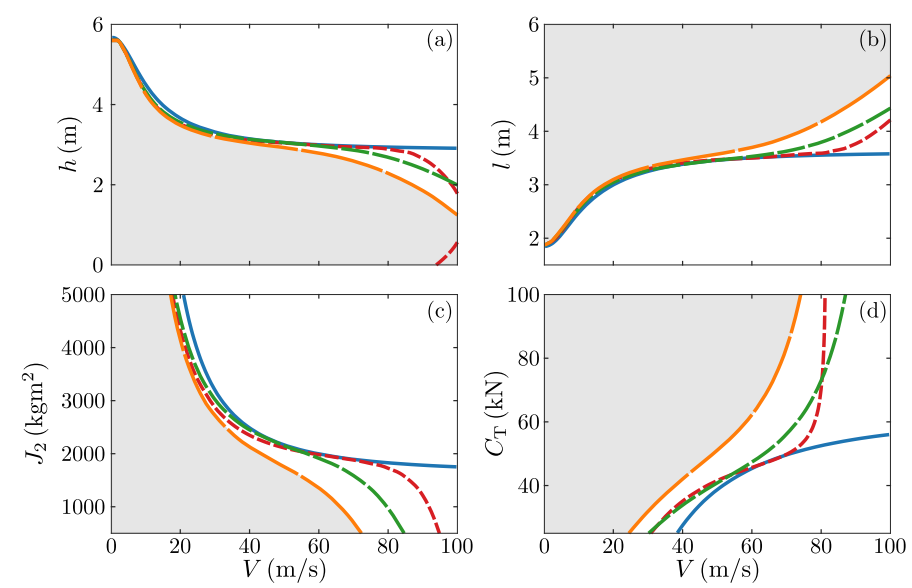

FIGURE 6. Stability charts of various trailer parameters and speed for the three different control laws: original lane keeping controller in red, Eqn. (19); additional feedback of trailer yaw angle in green, Eqn. (24); additional feedback of trailer yaw angle and yaw rate in orange, Eqn. (25). The shaded area shows the stable region for the third controller, while the open-loop stability boundary is plotted in blue. The time delay is set to $\tau=0.5 \mathrm{~s}$.

were not chosen to maximize the stable area but to provide a good comparison for a reasonable set of parameters.

\section{CONCLUSION}

The stability properties of a lane-keeping controller were analyzed in this paper when applied to a car-trailer system. It was shown that attaching a trailer to the car can reduce the linearly stable domain of control parameters, especially at higher speeds. In terms of trailer parameters, the controlled system shows similar results to the open-loop behavior of trailers, but feedback delay in the controller can destabilize originally stable parameter combinations. A possible solution for compensating the negative effects of feedback delay is the use of predictor control, such as finite spectrum assignment [26]. In the ideal case of no modeling and implementation errors, it is able to reproduce the linear stability properties of the delay-free system (with the drawback of higher computational cost).

In terms of trailer stability, no clear benefit was found when the trailer states are also utilized in the control law. Nevertheless, it is still recommended to adjust the control objective of the lanekeeping controller when a trailer is attached, to make sure that the trailer is also actively kept at the center of the lane.

Moreover, the largest difference between the different control approaches was observed at higher speed ranges, while the fixed control gains were tuned according to a relatively lower vehicle speed. The stable parameter domains could potentially be extended by applying a dynamic feedback controller that adapts to the current vehicle speed. Future continuation of this study involves exploring this possibility, as well as the validation of results with small-scale laboratory experiments.

\section{ACKNOWLEDGMENT}

This research was partly supported by the National Research, Development and Innovation Office under grant no. NKFI-128422 and by the Higher Education Excellence Program of the Ministry of Human Capacities in the frame of Artificial intelligence research area of Budapest University of Technology and Economics (BME FIKP-MI).

\section{REFERENCES}

[1] Olofsson, B., and Nielsen, L., 2020. "Using crash databases to predict effectiveness of new autonomous vehicle maneuvers for lane-departure injury reduction". IEEE Transactions on Intelligent Transportation Systems.

[2] Vlk, F., 1982. "Lateral dynamics of commercial vehicle combinations a literature survey". Vehicle System Dynamics, 11(5-6), pp. 305-324.

[3] Deng, W., and Kang, X., 2003. "Parametric study on vehicle-trailer dynamics for stability control". SAE transactions, pp. 1411-1419.

[4] Anderson, R., and Kurtz Jr, E., 1980. "Handlingcharacteristics simulations of car-trailer systems". SAE Transactions, pp. 2097-2113.

[5] Fratila, D., and Darling, J., 1996. "Simulation of coupled car and caravan handling behaviour". Vehicle System Dynamics, 26(6), pp. 397-429.

[6] Sharp, R., and Fernandez, M. A., 2002. "Car-caravan snaking: Part 1: The influence of pintle pin friction". Proceedings of the Institution of Mechanical Engineers, Part C: Journal of Mechanical Engineering Science, 216(7), pp. 707-722.

[7] Troger, H., and Zeman, K., 1984. "A nonlinear analysis of the generic types of loss of stability of the steady state motion of a tractor-semitrailer". Vehicle System Dynamics, 13(4), pp. 161-172.

[8] Hac, A., Fulk, D., and Chen, H., 2008. "Stability and control considerations of vehicle-trailer combination". SAE International Journal of Passenger Cars-Mechanical Systems, 1(2008-01-1228), pp. 925-937.

[9] Darling, J., Tilley, D., and Gao, B., 2009. "An experimental investigation of car-trailer high-speed stability". Proceedings of the Institution of Mechanical Engineers, Part D: Journal of Automobile Engineering, 223(4), pp. 471484.

[10] Kageyama, I., and Nagai, R., 1995. "Stabilization of passenger car-caravan combination using four wheel steering control". Vehicle System Dynamics, 24(4-5), pp. 313-327.

[11] Deng, W., Lee, Y. H., and Tian, M., 2004. "An integrated 
chassis control for vehicle-trailer stability and handling performance". SAE transactions, pp. 1041-1046.

[12] Fernandez, M. A., and Sharp, R., 2001. Caravan active braking system-effective stabilisation of snaking of combination vehicles. Tech. rep., SAE Technical Paper.

[13] Cheng, C., and Cebon, D., 2008. "Improving roll stability of articulated heavy vehicles using active semi-trailer steering”. Vehicle System Dynamics, 46(S1), pp. 373-388.

[14] Kimbrough, S., Elwell, M., and Chiu, C., 1994. "Braking controllers and steering controllers for combination vehicles". International Journal of Heavy Vehicle Systems, 1(2), pp. 195-223.

[15] Williams, J. M., and Mohn, F.-W., 2004. Trailer stabilization through active braking of the towing vehicle. Tech. rep., SAE Technical Paper.

[16] Greenwood, D. T., 2006. Advanced Dynamics. Cambridge University Press.

[17] Gantmacher, F., 1975. Lectures in Analytical Mechanics. MIR Publiser, Moscow.

[18] Beregi, S., Takács, D., and Stépán, G., 2016. "Tyre induced vibrations of the car-trailer system". Journal of Sound and Vibration, 362, pp. 214-227.

[19] Pacejka, H. B., 2006. Tyre and Vehicle Dynamics. Butterworth-Heinemann, Oxford.

[20] Stépán, G., 1989. Retarded Dynamical Systems: Stability and Characteristic Functions. Longman Scientific \& Technical.

[21] Michiels, W., and Niculescu, S.-I., 2007. Stability and Stabilization of Time-Delay Systems. Society for Industrial \& Applied Mathematics, U.S.

[22] Insperger, T., and Stépán, G., 2011. Semi-Discretization for Time-Delay Systems. Springer.

[23] Bachrathy, D., and Stépán, G., 2012. "Bisection method in higher dimensions and the efficiency number". Periodica Polytechnica Mechanical Engineering, 56(2), pp. 81-86.

[24] Vörös, I., Várszegi, B., and Takács, D., 2019. "Lane keeping control using finite spectrum assignment with modeling errors". Dynamic Systems and Control Conference, ASME. V003T18A002.

[25] Niculescu, S.-I., Gu, K., and Abdallah, C. T., 2003. "Some remarks on the delay stabilizing effect in siso systems". In Proceedings of the 2003 American Control Conference, 2003., Vol. 3, IEEE, pp. 2670-2675.

[26] Manitius, A. Z., and Olbrot, A. W., 1979. "Finite spectrum assignment problem for systems with delays". IEEE Transactions on Automatic Control, 24(4), pp. 541-553. 